\title{
Éditorial
}

\section{La chirurgie orale sauvera-t-elle la médecine orale ?}

\author{
Jean-Christophe Fricain
}

La spécialité de chirurgie orale a vu le jour en 2011 et la première promotion d'internes est sortie en 2015 . Je ne reviendrai pas sur l'historique de la spécialité, elle a été très précisément détaillée par le Professeur Benoît Lefèvre dans l'éditorial du numéro un de MBCB 2016 [1]. En revanche, je profiterai de cet éditorial pour aborder la position de la médecine orale au sein de la spécialité et sa visibilité en France et dans le monde.

Récemment, Crispian Scully a publié un article intitulé «Oral medicine (stomatology) across the globe : birth, growth, and future » [2], où il fait un état des lieux de la médecine orale. La médecine orale est définie par un champ de compétences qui recouvre le diagnostic et le traitement non chirurgical des maladies et lésions oro-faciales d'origine locale ou générale. Cette définition précise donc qu'il s'agit d'une spécialité non chirurgicale, ce qui semble l'opposer à la chirurgie orale. Cette opposition entre la médecine et la chirurgie n'a bien sûr aucun sens en pratique clinique où nombre de lésions buccales nécessitent un traitement médico-chirurgical. S'il apparaît donc clair que médecine et chirurgie orales sont complémentaires, il n'en demeure pas moins que la place de la médecine orale en France est confuse et peu visible aujourd'hui. Plusieurs raisons, pédagogiques, scientifiques et cliniques expliquent cette nébuleuse :

- L'enseignement de la médecine orale fait partie intégrante du programme de formation approfondi en sciences odontologiques. Ainsi, tous les chirurgiens dentistes diplômés ont pu bénéficier d'un enseignement spécifique. Force est de constater que malgré cet enseignement, peu de chirurgiens dentistes pratiquent la médecine orale. Dans le cadre de la formation continue, il existe un Diplôme d'Université dispensé par l'Université Paris Descartes (Dermatologie-vénérologie de la muqueuse buccale) et un Diplôme Inter Universitaire entre les Universités Pierre et Marie Curie à Paris et François Rabelais de Tours (Pathologies de la muqueuse buccale). Cette offre de formation continue est complétée par des actions ponctuelles de formation financées ou non par l'Organisme Gestionnaire du
Développement Professionnel Continu (OGDPC). Toutes ces formations ne recouvrent qu'une partie de la médecine orale : les pathologies osseuses ainsi que les douleurs chroniques en sont souvent exclues. Il n'existe à ce jour aucun Master de médecine orale en France. La formation la plus complète théorique et pratique en médecine orale est dispensée dans le Diplôme d'Etudes Spécialisées en Chirurgie Orale (DESCO). Les domaines de compétence du chirurgien oral étant sur le plan médical : la dermatologie buccale, le dépistage des lésions et tumeurs malignes de la cavité buccale, des maxillaires et de la mandibule, les douleurs oro-faciales, les pathologies de l'appareil manducateur, les pathologies des glandes salivaires. - Sur le plan scientifique, il n'existe aucune société scientifique dédiée spécifiquement à la médecine orale [2]. Au moins 4 sociétés scientifiques s'intéressent en partie à la médecine orale : la Société Française de Chirurgie Orale (SFCO) , la Société Française de Stomatologie Chirurgie Maxillo-Faciale et Chirurgie Orale (SFSCMFCO), la Société Française de Dermatologie (SFD), la Société Française d'Oto-Rhino-Laryngologie et de chirurgie de la face et du cou (SFORL). Toutes ces sociétés sont affiliées à une revue qui publie des articles de médecine orale. La revue Médecine Buccale Chirurgie Buccale est la seule revue qui publie essentiellement des articles de médecine orale. Sa visibilité internationale reste limitée du fait de la langue de publication (français). Sa publication en anglais permettrait d'accroître sa visibilité internationale. Au niveau international, au moins 4 sociétés sont associées à une revue : la European Association of Oral Medicine et l'Oral Medicine Academy of Australia avec Oral Diseases ; l'American Association of Oral Medicine avec Oral Surgery, Oral Medicine, Oral Pathology, Oral Radiology et l'Ibero-American Academy of Oral Pathology and Medicine avec Medicina Oral Patología Oral y Cirugía Bucal.

- Sur le plan de l'exercice professionnel, en France, la médecine orale était historiquement pratiquée en majorité par les stomatologues qui ont le plus souvent un exercice médicochirurgical alors que les chirurgiens maxillo-faciaux ont

\footnotetext{
* Correspondance : fricainj@aol.com
}

This is an Open Access article distributed under the terms of the Creative Commons Attribution License (http://creativecommons.org/licenses/by/4.0), which permits unrestricted use, distribution, and reproduction in any medium, provided the original work is properly cited 
principalement un exercice chirurgical. D'autres professionnels de santé peuvent être consultés en fonction des symptômes : dentiste, médecin généraliste, neurologue, $0 R L$, dermatologue. Du fait de la disparition progressive des stomatologues et de leur remplacement par les chirurgiens oraux, il apparaît logique que les chirurgiens oraux utilisent ce champ de compétence dans leur exercice. Cependant de nombreux écueils subsistent :

- La dénomination chirurgie orale n'est pas lisible en terme de médecine orale,

- La chirurgie orale est un DES de chirurgie et non de médecine, choisi dans la filière chirurgie par les étudiants à l'examen national classant,

- la rémunération des consultations est largement inférieure aux actes chirurgicaux.

Enfin nul ne doute que certains des spécialistes qualifiés en médecine bucco-dentaire pratiqueront la médecine orale. Alors, la chirurgie orale sauvera-t-elle la médecine orale? Il faut le souhaiter car nombre de pathologies buccales sont mal connues des praticiens de première ligne (médecins généralistes et dentistes). Il en découle une augmentation des délais diagnostiques, et la prescription de nombreux traitements inadaptés. Le réseau naturel devra être l'adressage du patient au chirurgien oral. Cependant, le risque de voir un certain nombre de chirurgiens oraux se détourner de la pratique médicale au profit de la chirurgie n'est pas nul. Ainsi, je voudrais par cet éditorial inviter tous les chirurgiens oraux à exercer pleinement dans la totalité de leur champ de compétence et à le faire savoir.

Conflits d'intérêt : aucun

\section{Références}

1. B Lefevre. La chirurgie orale : discipline hospitalo-universitaire et spécialité. Bref historique. Med Buccale, Chir Bucccale 2016; 22:3-5.

2. Scully C, Miller CS, Aguirre Urizar JM, Alajbeg I, et al. Oral medicine (stomatology) across the globe: birth, growth, and future. Oral Surg Oral Med Oral Pathol Oral Radiol 2016;121: 149-157. 